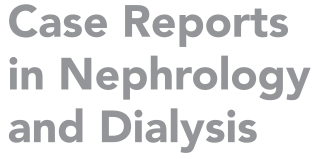

Case Reports and Dialysis

\title{
Hemolysis in a Patient during Hemodialysis
}

\author{
Maxime Taghavi Lucas Jacobs Saleh Kaysi \\ Maria do Carmo Filomena Mesquita \\ Department of Internal Medicine, Nephrology Clinic, CHU Brugmann, Université Libre de \\ Bruxelles, Brussels, Belgium
}

\author{
Keywords \\ Hemolysis $\cdot$ Hemodialysis $\cdot$ Faulty blood tubing sets $\cdot$ Kinking of bloodlines
}

\begin{abstract}
We report a case of hemolysis during a hemodialysis (HD) session in a 71-year-old man. His end-stage kidney disease is secondary to light-chain amyloidosis with renal involvement. Despite immunosuppressive treatment, his renal function continued to decline, and dialysis had to be initiated. Peritoneal dialysis (PD) was started but that had to be converted to HD because of pleural effusion due to PD fluid leakage. On the event day, the patient presented a respiratory distress $2 \mathrm{~h}$ after the initiation of HD. He developed a sudden onset of dyspnea with hypoxemia, associated with abdominal pain, nausea, and vomiting. He also presented chest pain with arterial hypertension. The pre-pump arterial and post-pump pressures were, respectively, 40 and $100 \mathrm{~mm} \mathrm{Hg}$, with no machine alarm. The blood color in the circuit changed and became darker, so HD was stopped immediately without blood restitution, and then a blood workup was obtained, and the patient was treated with oxygen therapy, IV methylprednisolone $40 \mathrm{mg}$, and IV furosemide $100 \mathrm{mg}$. Tubing checkup performed after the incident showed a kinked arterial tube which led to the suspicion of acute hemolysis. Blood transfusion was therefore urgently ordered, and the patient was immediately transferred to the intensive care unit (ICU). Artificial ventilation was required for 4 days, with initial massive blood transfusion. A 24-h treatment with extracorporeal cytokine adsorber CytoSorb ${ }^{\circledR}$ was also performed, followed by the regular HD sessions thrice weekly. Evolution was favorable, and the patient was discharged from the ICU 18 days later.
\end{abstract}

\section{Karger ${ }^{\prime \prime}=$}




\section{Introduction}

In 2015, according to the European Renal Association- European Dialysis and Transplant Association (ERA-EDTA) Registry, 81,373 individuals commenced renal replacement therapy for end-stage kidney disease in Europe, representing an incidence rate of 119 per million population [1]. Hemolysis or premature breakdown of erythrocytes, associated with hemodialysis (HD) is rarely reported, being nowadays a rare entity, but it remains a potential lifethreatening condition associated with a significant morbidity and mortality. Stringent national and international standards, technological advancements in the membrane and dialysis equipment design, dialyzer purification methods, and water treatment systems have greatly reduced the incidence of hemolysis [2]. Here, we report the investigations and the management of acute hemolysis that occurred in one of our patients during an HD session.

\section{Case Report}

Here, we report the case of a 71-year-old man, with end-stage kidney disease on maintenance HD. Medical history included a light-chain amyloidosis with renal and cardiac involvement, treated with bortezomib, cyclophosphamide, and dexamethasone with a complete hematological response, but renal function continued to decline, and renal replacement therapy was initiated in 2017. Medical history also included type 2 diabetes mellitus, arterial hypertension, sleep apnea, and a cerebral transient ischemic attack in 2014.

His treatment consisted of once daily doses of $100 \mathrm{mg}$ allopurinol, $5 \mathrm{mg}$ amlodipine, $10 \mathrm{mg}$ perindopril, $10 \mathrm{mg}$ bisoprolol, $1 \mathrm{~g}$ sodium bicarbonate, and $20 \mathrm{mg}$ pantoprazole. On nondialysis days, he had once daily doses of $125 \mathrm{mg}$ furosemide and $15 \mathrm{~g}$ of calcium Kayexalate.

On the event day, $2 \mathrm{~h}$ after the initiation of HD, he developed sudden dyspnea with hypoxemia and chest pain, associated with abdominal pain, nausea, and vomiting. Ultrafiltration volume planned was 1,400 $\mathrm{mL}$, and the patient was $1 \mathrm{~kg}$ higher than the dry weight. The pre-pump arterial and post-pump pressures were, respectively, 40 and $100 \mathrm{~mm} \mathrm{Hg}$ with no machine alarm. Patient's vascular access consisted in a radio-cephalic arteriovenous fistula. An arteriovenous fistula was needled with $217 \mathrm{G}$ needles, and the blood flow rate was $350 \mathrm{~mL} / \mathrm{min}$, and the dialysate temperature was $36^{\circ} \mathrm{C}$.

A blood workup was obtained, and the patient was treated with oxygen therapy, IV methylprednisolone $40 \mathrm{mg}$, and IV furosemide $100 \mathrm{mg}$. HD was stopped immediately without blood restitution.

Tubing checkup performed after the incident showed a kinked arterial tube which led to the suspicion of acute hemolysis. Blood transfusion was therefore urgently ordered, and the patient was immediately transferred to the intensive care unit (ICU).

Physical examination at the ICU revealed arterial hypertension (blood pressure of $220 / 100 \mathrm{~mm} \mathrm{Hg}$ ) and pulmonary overload with wet crackles. His laboratory findings showed acute hemolytic anemia with a hemoglobin level of $2.2 \mathrm{~g} / \mathrm{dL}(N 13-18 \mathrm{~g} / \mathrm{dL})$, whereas it was $9.5 \mathrm{~g} / \mathrm{dL}$ on the same day. Haptoglobin was $<10 \mathrm{mg} / \mathrm{dL}(N 30-200 \mathrm{mg} / \mathrm{dL})$. Lactate dehydrogenase increased from 219 to 8,761 UI/L ( $N 135-225 \mathrm{UI} / \mathrm{L})$. The aspartate transaminase level increased from $10 \mathrm{UI} / \mathrm{L}$ to 5,041 UI/L $(N<41 \mathrm{UI} / \mathrm{L})$, and creatine phosphokinase increased from 54 to 6,483 UI/L $(N<190 \mathrm{UI} / \mathrm{L})$. Bilirubin increased from 0.3 to $11.9 \mathrm{mg} / \mathrm{dL}(N$ 0.2-1.2 $\mathrm{mg} / \mathrm{dL}$ ). The potassium level was also increased to $6.2 \mathrm{mmol} / \mathrm{L}$, which was unusual for the patient ( $N$ 3.5-4.8 mmol/L) (Fig. 1).

Massive hemolysis was also complicated with disseminated intravascular coagulation with low fibrinogen and platelet $(50 \mathrm{mg} / \mathrm{dL}[N 160-415 \mathrm{mg} / \mathrm{dL}]$ and $43.000 / \mu \mathrm{L}[N 150-440.000 / \mu \mathrm{L}]$,

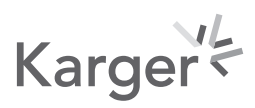


Case Reports in Nephrology and Dialysis
Case Rep Nephrol Dial 2021;11:348-354

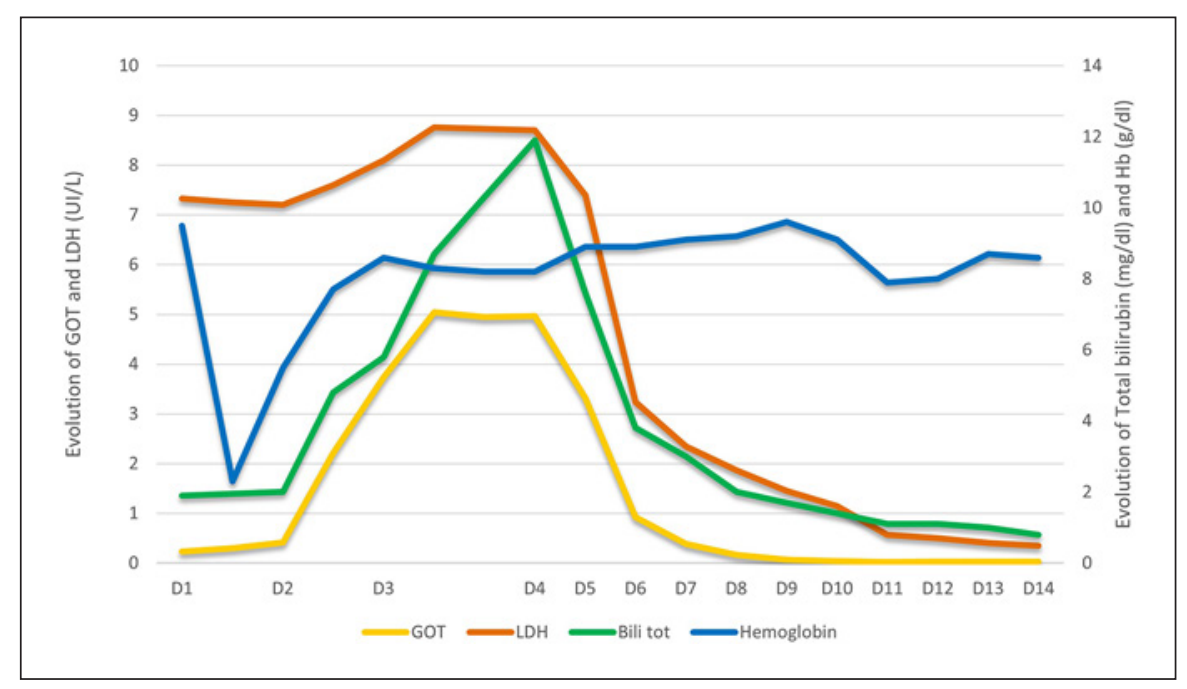

Fig. 1. Biological values during 14 days in the ICU. Left ordinate: GOT (UI/L) and LDH (UI/L); Right ordinate: Bili tot (mg/dL) and $\mathrm{Hb}(\mathrm{g} / \mathrm{dL}$ ). GOT, glutamic oxaloacetic transaminase; LDH, lactate dehydrogenase; Bili tot, total bilirubin; $\mathrm{Hb}$, hemoglobin.

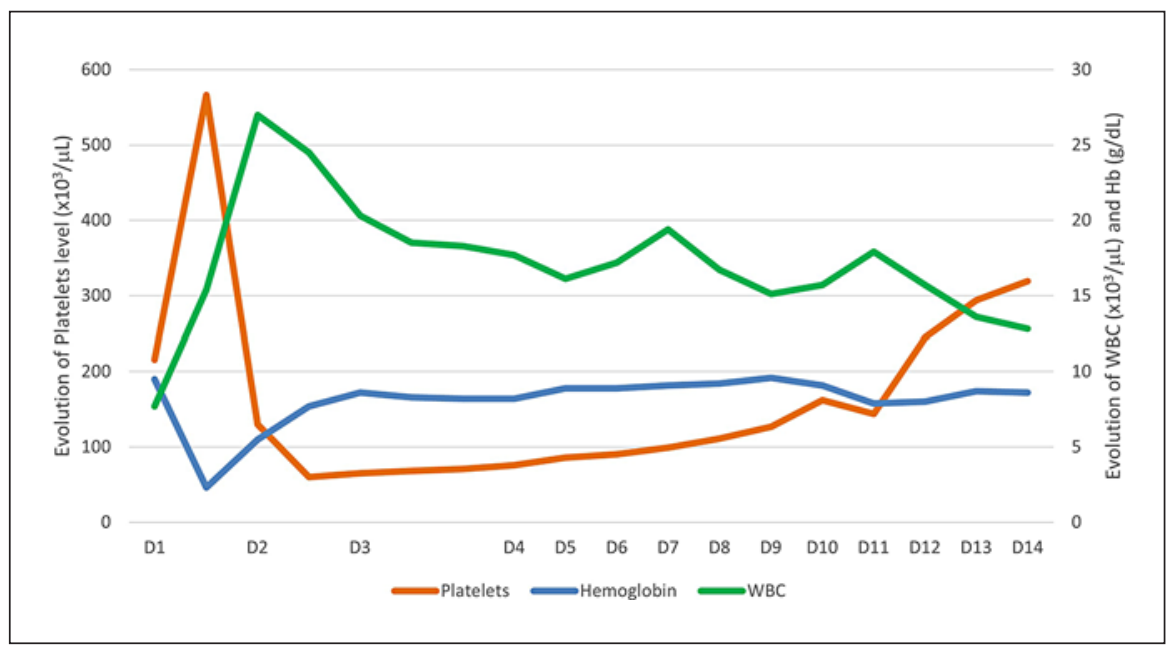

Fig. 2. Hematological values during 14 days in the ICU. Left ordinate: platelets and thrombocytes count $\left(\times 10^{3} / \mu \mathrm{L}\right)$; Right ordinate: WBC $\left(\times 10^{3} / \mu \mathrm{L}\right) ; \mathrm{Hb}(\mathrm{g} / \mathrm{dL})$. WBC, white blood cell count; Hb, hemoglobin.

respectively) associated with rectorrhagia. No thrombotic manifestation was observed. Hematological variations during ICU care are shown in Figure 2.

Endotracheal intubation and artificial ventilation were required for 4 days, with initial massive blood transfusion. A 24-h treatment with an extracorporeal cytokine adsorber CytoSorb ${ }^{\circledR}$ was also performed at the ICU, in order to reduce free hemoglobin (fHb) and other erythrocytes and cytoplasmic molecules in the bloodstream. Regular HD sessions were continued.

During the ICU stay, he remained very confused during several days. He was discharged from the ICU 18 days later and sent to a rehabilitation center. Meanwhile, the patient was seen thrice weekly during his regular dialysis sessions, and his physical and mental status improved gradually. A mini-mental test was done recently, and he scored 26/30. 
Fig. 3. Showing the kinked faulty arterial bloodline tubing during the HD session. HD, hemodialysis.

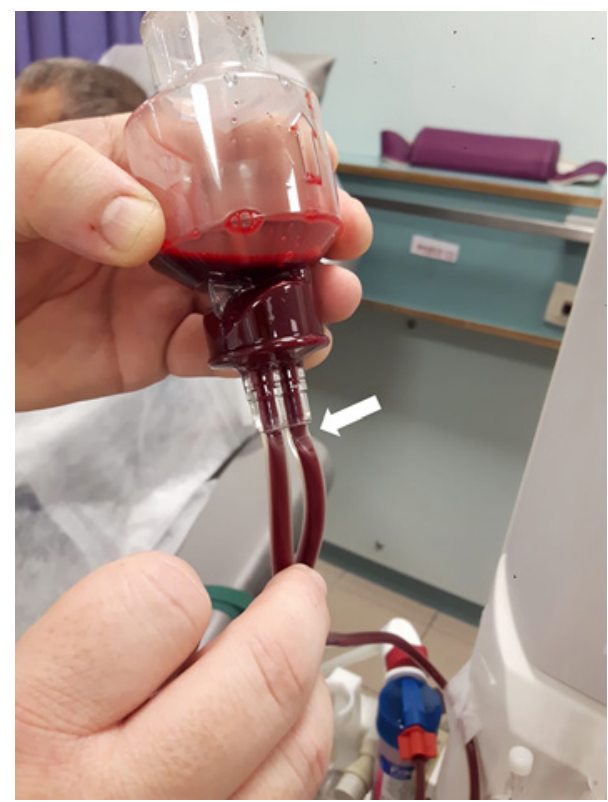

\section{Discussion}

Our patient presented massive hemolysis due to post-pump arterial tube kinking. This complication is nowadays extremely rare, and only few cases are described in the literature, and some outbreaks have been reported. The most important one took place in 1998 and was associated to the use of the same faulty lot of disposable tubing sets from one manufacturer [3,4]. Thirty patients were involved in the states of Nebraska, Maryland, and Massachusetts, and 3 of them died.

Early recognition of hemolysis in HD is mandatory to minimize morbidity and mortality. Also, implications of such findings are important to avoid recurrence by contacting the manufacturer, recalling the faulty tubing sets [4]. Indeed, in our case, after checking for all the same manufacturer's sets, we found multiple kinked tubes (Fig. 3).

Clinicians should be aware that when kinking occurs just after the pump segment (e.g., at the dialyzer inlet port), it causes back pressure elevation but no alarm [5]. While post-pump obstructions and hemolysis can occur without causing noticeable changes to the pre-pump arterial and venous blood line pressures (due to the highly occlusive setting of the roller blood pump), recognizing sudden and/or sustained decreases in the circuit pressures during treatment may help to prevent adverse patient events [6].

Clinical manifestations are mostly nonspecific such as nausea, abdominal pain, back pain, headache, or malaise [5] and depend on the severity of hemolysis. Abdominal pain and hypertension seem to be the most frequent features and represented, respectively, 60 and $66 \%$ on a 30-patient case study [4]. Hemolysis can also lead to acute pancreatitis [7]. Sudden deepening of pigmentation with deep erythema have also been described [8]. In severe cases, patients can experience cardiorespiratory failure, requiring immediate ICU management [3, 4]. Our patient presented with classical severe symptoms, and blood color changes were observed in the tubing. HD session was immediately stopped without restitution.

Outcomes vary from the need for blood transfusion to life-threatening complications from anemia, hyperkalemia, plasma hemoglobin-induced vasoconstriction, and acute pancreatitis $[6,7]$. To reduce plasma $\mathrm{fHb}$, and therefore limit hemolysis complications, such as smooth muscle dystonia, vasculopathy, and endothelial dysfunction [9], we performed a 24-h treatment with extracorporeal cytokine adsorber CytoSorb ${ }^{\circledR}$. The latter is associated with a 
significant lowering in plasma $\mathrm{fHb}$ in some studies during cardiopulmonary bypass-induced hemolysis [10]. Whether lower $\mathrm{fHb}$ is associated or not with better outcomes is unclear. Unfortunately, we were not able to measure the fHb level.

Differential diagnosis can be fastidious since causes are numerous. In a normal setting, red blood cells are exposed to shear stress because of a differential force between the center and the wall of the extracorporeal circuit [11]. Common causes are chemical contamination by oxidizing agents (e.g., copper, zinc, chloramine, or nitrate contamination of the dialysate), reducing agents (e.g., formaldehyde used to disinfect reprocessed dialyzers or water treatment systems) [12], excessive dialysis solution heat, inadvertent HD against distilled water, mechanical injury of erythrocytes from maloccluded blood pumps, arterial line collapse, kinked or obstructed HD tubing, single-needle high-flow dialysis, or partial occlusion of the HD catheter $[2,13]$. Mechanical hemolysis related to the use of tandem dialyzers has been described [14] as well as excessive pressure in the line between the arterial blood pump and the dialyzer [8]. Malposition of the needle in the fistula [15] and mechanical thrombectomy of the fistula [16] causing massive hemolysis have also been reported. Uremia and oxidative stress are also implicated, mostly at the initiation of dialysis [2].

Our investigation traced the hemolysis to a partially occluded HD blood tubing produced by a single manufacturer. The manufacturer issued a voluntary nationwide recall of the lots of implicated HD blood tubing cartridge sets.

Attention to decrease HD circuit pressures indicating post-pump tubing kinks is important [6] as well as re-evaluation of pre-pump arterial pressure to avoid inadequate dialysis and hemolysis [17]. In this case, as the defects on the tubing sets were not very prominent, it escaped the attention of the nursing staff.

\section{Conclusions}

Kinking of bloodlines should be known by HD center's health worker as an acute complication in HD patients. Tubing checkups by nurses should be systematic and should be repeated after initiation of each HD session. Monitoring of the patient and dialyzer's parameters by nurses is of a great importance since a sudden and/or sustained decreases in the circuit pressures during treatment may be suggestive, even in the absence of an alarm. In case of hemolysis, circuit's blood should not be returned to the patient. In life-threatening situations, hospitalization in the ICU is required, and treatment with CytoSorb can be proposed, but its efficacy must be demonstrated. The detection of a faulty dialysis set should lead to an immediate communication with the manufacturer and the hospital authorities in order to prevent other cases. As such events are extremely rare, we recommend regular training sessions discussing acute complications of HD for medical and the nursing staff.

\section{Acknowledgments}

The authors acknowledge each and every clinician and the nursing staff who treated and cared for the patient described in this case report.

\section{Statement of Ethics}

This retrospective review of patient data did not require ethical approval in accordance with local guidelines. This article does not contain any studies with human participants. This is an anonymous case presentation of an HD patient. Written informed consent was obtained from the patient for publication of this case report and any accompanying images.

\section{Karger'}




\section{Conflict of Interest Statement}

The authors have no conflicts of interest to declare.

\section{Funding Sources}

The authors received no funding for this work.

\section{Author Contributions}

M.T. and M.C.F.M. developed the manuscript idea and drafted the first version. L.J. did the data collection and figures. M.T. and M.C.F.M. participated in drafting of the discussion of the manuscript, and S.K. took part in revising the article critically. All the authors read and approved the final manuscript.

\section{Data Availability Statement}

All data supporting this work are included in this article. Any further inquiries can be directed to the corresponding author.

\section{References}

1 Kramer A, Pippias M, Noordzij M, Stel VS, Afentakis N, Ambühl PM, et al. The European Renal Association - European dialysis and transplant association (ERA-EDTA) registry annual report 2015: a summary. Clin Kidney J. 2018 Feb; 11(1):108-22.

2 Tharmaraj D, Kerr PG. Haemolysis in haemodialysis. Nephrology. 2017;22(11):838-47.

3 Centers for Disease Control and Prevention (CDC). Multistate outbreak of hemolysis in hemodialysis patients: Nebraska and Maryland, 1998. MMWR Morb Mortal Wkly Rep. 1998 Jun 19;47(23):483-4.

4 Duffy R, Tomashek K, Spangenberg M, Spry L, Dwyer D, Safranek TJ, et al. Multistate outbreak of hemolysis in hemodialysis patients traced to faulty blood tubing sets. Kidney Int. 2000 Apr;57(4):1668-74.

5 Gault MH, Duffett S, Purchase L, Murphy J. Hemodialysis intravascular hemolysis and kinked blood lines. Nephron. 1992;62(3):267-71.

6 Malinauskas RA. Decreased hemodialysis circuit pressures indicating postpump tubing kinks: a retrospective investigation of hemolysis in five patients. Hemodial Int. 2008;12(3):383-93.

7 Abtahi M, Uzan M, Souid M. Hemolysis-induced acute pancreatitis secondary to kinked hemodialysis blood lines. Hemodial Int. 2007;11(1):38-41.

8 Seukeran D, Fletcher S, Sellars L, Vestey JP. Sudden deepening of pigmentation during haemodialysis due to severe haemolysis. Br J Dermatol. 1997;137(6):997-9.

9 Rother RP, Bell L, Hillmen P, Gladwin MT. The clinical sequelae of intravascular hemolysis and extracellular plasma hemoglobin: a novel mechanism of human disease. JAMA. 2005;293(13):1653-62.

10 Gleason TG, Argenziano M, Bavaria JE, Kane LC, Coselli JS, Engelman RM, et al. Hemoadsorption to reduce plasma-free hemoglobin during cardiac surgery: results of REFRESH I pilot study. Semin Thorac Cardiovasc Surg. 2019;31(4):783-93.

11 Sweet SJ, McCarthy S, Steingart R, Callahan T. Hemolytic reactions mechanically induced by kinked hemodialysis lines. Am J Kidney Dis. 1996;27(2):262-6.

12 Kitching AR, Ritchie D, Wong JK, May A, Hatfield PJ. Chloramine-induced hemolysis associated with neurological symptoms in a home hemodialysis patient. Clin Nephrol. 2001;55(3):259-60.

13 Saha M, Allon M. Diagnosis, treatment, and prevention of hemodialysis emergencies. Clin J Am Soc Nephrol. 2017;12(2):357-69.

14 Kazmi A, Canada R, Wall BM. Mechanical haemolysis related to the use of tandem dialyzers. NDT Plus. 2008; 1(2):89-91.

15 Yoon J, Thapa S, Chow RD, Jaar BG. Hemolysis as a rare but potentially life-threatening complication of hemodialysis: a case report. BMC Res Notes. 2014;7:475. 
16 Carrera LA, Reddy R, Pamoukian VN, Michelis MF, DeVita MV, Rosenstock J. Massive intravascular hemolysis with mechanical rheolytic thrombectomy of a hemodialysis arteriovenous fistula. Semin Dial. 2013;26(1):E5-7.

17 Shibata E, Nagai K, Takeuchi R, Noda Y, Makino T, Chikata Y, et al. Re-evaluation of pre-pump arterial pressure to avoid inadequate dialysis and hemolysis: importance of prepump arterial pressure monitoring in hemodialysis patients. Artif Organs. 2015 Jul;39(7):627-34. 